\title{
Investigation of the Effect of Vertimax V8 Elastic Resistance Platform and Classic Strength Training on Dynamic Balance in Basketball Players
}

\author{
Oguzhan Yuksel ${ }^{1}$, Mustafa S. Erzeybek ${ }^{1}$, Fatih Kaya ${ }^{2} \&$ Sadettin Kirazci $^{3}$ \\ ${ }^{1}$ School of Physical Education and Sports, Kutahya Dumlupinar University, Kutahya, Turkey \\ ${ }^{2}$ School of Physical Education and Sports, Erzincan Binali Yildirim University, Erzincan, Turkey \\ ${ }^{3}$ Department of Physical Education and Sports, Middle East Technical University, Ankara, Turkey \\ Correspondence: Oguzhan Yuksel, School of Physical Education and Sports, Kutahya Dumlupinar University, \\ Kutahya, Turkey. E-mail: oguzhan.yuksel@dpu.edu.tr
}

Received: January 16, 2019

Accepted: February 15, 2019 Online Published: March 6, 2019

doi:10.5539/jel.v8n2p188

URL: https://doi.org/10.5539/jel.v8n2p188

\begin{abstract}
This study aimed to investigate the effects on the dynamic balance of two different resistance training in male basketball players. 25 male basketball players who were over 18 participated in the study voluntarily. Participants were divided into two groups by using random sampling method as VertiMax strength training group and traditional strength training group (VertiMax, $n=13$; classic, $n=12$ ). Both training programs were administered 3 times a day for 6 weeks. In standing position, the dynamic balance values recorded on the right and left foot for 60 seconds were assessed by using EasyTech Libra Oscillating balance board. The scores used in the assessments were IBP (index of balance precision), recovery time right (return time of the visual helix balance from right side), and recovery time left (return time of the visual helix balance from left side). All tests were performed with visual feedback. Statistical analysis used three-factor mixed ANOVA. At the end of the training period, IBP (index of balance precision), recovery time right (return time of the visual helix balance from right side), recovery time left (return time of the visual helix balance from left side) showed that dynamic balance scores were similar in the VertiMax and classic training groups for right and left feet. VertiMax group in dynamic balance, the changes in IBP and recovery time right scores were found to be better. In both training groups, when the recovery time right values of the right and left feet were compared at the end of the process, it was seen that the rotation time from the right side of the visual helix motion area of the left leg was shorter $(\mathrm{p}<0.05)$. The results show that the VertiMax strength training included in the pre-season exercises positively affects participants' balance performance.
\end{abstract}

Keywords: dynamic balance, basketball, VertiMax training

\section{Introduction}

Basketball is a sport that requires speed, agility, upper and lower body strength, maximal aerobics and aerobic resistance (Borowski et al., 2008). During the game, it is seen that different kinds of activity and movement patterns, fast and frequent runs, deflections and jumps are displayed every 2 seconds during the game (McInnes et al.,1995).

In basketball, different approaches to balance as well as biomotor abilities should be taken into consideration, because balance is among the most important coordination skills for basketball (Kostopoulos et al., 2012).

Balance is usually an effective component of athletic activity. Therefore, besides the possible decrease in injuries, it can increase athletic performance with the support of neuromuscular training programs and support the improvement of motor control (McLeod et al., 2009). Balance is an indispensable part of many actions and affects movement ability. Balance control is a complex motor ability that entails planning and performing flexible movement forms as well as the integration of sensory inputs (Ferdjallah et al., 2002). It has a considerable impact on performance in sports activities. Balance also contributes to the prevention of injuries and rehabilitation process. The measurement of balance is effective in the clinical assessment of locomotor functions (Hahn et al., 1999). 
In basketball, strength is a dominant biomotor ability in competitions. The ability to generate lower body explosive power is accepted as an important factor in sports activities and can be considered as the expression of the amount of performance displayed in a specific time period (Kaya et al., 2018). Speed and strength stand out in change of direction, acceleration, jump and sprint performances in many sportive actions (Holcomb et al., 1996). Although specific resistance training is important, general strength training increases body mass and contribute to the decrease of injury risk and developing core stability. Hypertrophy and general strength exercises can enhance performance, but specific exercise programs should be preferred for optimal transfer from training (Young, 2006). In addition to general strength training, plyometric training is also widely used as a common characteristic of team plays (Shiner et al., 2005). Besides, it has been indicated that strength training elicits a significant increase in static and dynamic balance (Perrin et al., 1999; Paterno \& Myer, 2004; Warren et al., 2010). In their study to investigate whether static and dynamic balance vary across different sports, Bressel et al. (2007) found that static and dynamic balance scores did not differ in gymnasts and soccer players, however basketball players displayed lower static balance compared with gymnasts and lower dynamic balance compared with soccer players Bressel et al. (2007).

It is reported that VertiMax training model that provides resistance as a specific strength exercise to improve lower body power and vertical jump performance also has the capability to generate power via resisted shoulder flexion during jumps by creating resistance (Carlson et al., 2009). In their study comparing the VertiMax training model and traditional models for power and conditioning, Rhea et al. state that the Vertimax training model increases vertical jump and lower extremity power (Rhea et al., 2008).

The aim of this study was to examine the effect of traditional strength training and loaded jump training using the VertiMax on dynamic balance.

\section{Method}

\subsection{Study Group}

25 male basketball players who were over 18 participated in the study voluntarily. The athletes were randomly divided into two groups: traditional strength training group (classic, $\mathrm{n}=13$ ) and VertiMax training group (VertiMax, $\mathrm{n}=12$ ). Height, weight and age of the basketball players in the VertiMax group was as follows: $191.77 \pm 9.23 \mathrm{~cm}, 92.38 \pm 12.04 \mathrm{~kg}$, and $22.62 \pm 1.45$ years while the height, weight and age of the athletes in the classic group were $190.88 \pm 9.32 \mathrm{~cm}, 90.4 \pm 11.71 \mathrm{~kg}$, and $22.84 \pm 1.46$ years, respectively. All of the participants volunteered to take part in the study and training groups were selected through random sampling method.

The day before the assessments, the participants were informed about the test and were told that the tests would cause no problems for their health. The study implemented pre-test and post-test protocol. Pre-test assessments were performed 72 hours before initiating the training protocols, and after that, training protocols were implemented. During training, no measurement was taken. Post-test measurements were performed within the week when the training protocol was completed. The participants attended the training protocol on a regular basis and none of them missed a training session.

The participants were asked to continue their routine diet throughout the study, and no participant took ergogenic aids that could affect training and assessment results or cause a change in performance. 48 hours before test days, the participants stopped to take food or liquid supplements containing alcohol, caffeine or intense stimulants. While all the participants had basketball background, they followed only the specified training protocol throughout the study.

\subsection{Assessment of Dynamic Balance}

To measure dynamic balance stability, the Libra oscillating balancing board manufactured by EasyTech (a computer set placed on a platform with $43 \mathrm{~cm}$ length, $42 \mathrm{~cm}$ width and $65 \mathrm{~cm}$ height) was used. The testing stand comprised two elements. The system is completed by connecting the balancing board to EasyTech 2.2001-2.0 computer software with a USB interface. EasyTech 2.2001-2.0 software interface computes four parameters depending on swing in a balanced position (Figure 1). Total Area: the area within the movement path line that the subject maintains balance. External Area: the area outside the movement path line that the subject is not on the balance line. External Time: the period of time when the subject is outside the balance line. Recovery Time: the period of time when the subject is outside the balance line area and returns from right and left sides back to the balance line.

Index of balancing precision $(\mathrm{IBP})=(\mathrm{EA} / \mathrm{TA}) * 100 .($ External area $=\sqrt{ }$ external area right $+\sqrt{ }$ external area left $)$ (Total area $=\sqrt{ }$ total area right $+\sqrt{ }$ total area left) (Tchórzewski et al., 2013). As is seen in the screenshot of the 
EasyTech Libra in Figure 1, the subject's attempt to stabilise the balancing board within the helix area is accepted as balance precision.

The bottom surface of the Libra balancing board contains an adjustable plastic apparatus with difficulty levels of $10 \mathrm{~cm}, 25 \mathrm{~cm}$ and $40 \mathrm{~cm}$. For the participants of this study, the $40 \mathrm{~cm}$ apparatus was used. Standing on the balancing board with their right foot at the centre, each subject started the test on the Libra oscillating balancing board with right foot. After 3 trials, a 5-minute break was taken, and then, 3 more trials were performed with the left foot. Balance scores, right-left total area, right-left external area, right-left recovery time and right-left external time values were recorded (Tchórzewski et al., 2010).

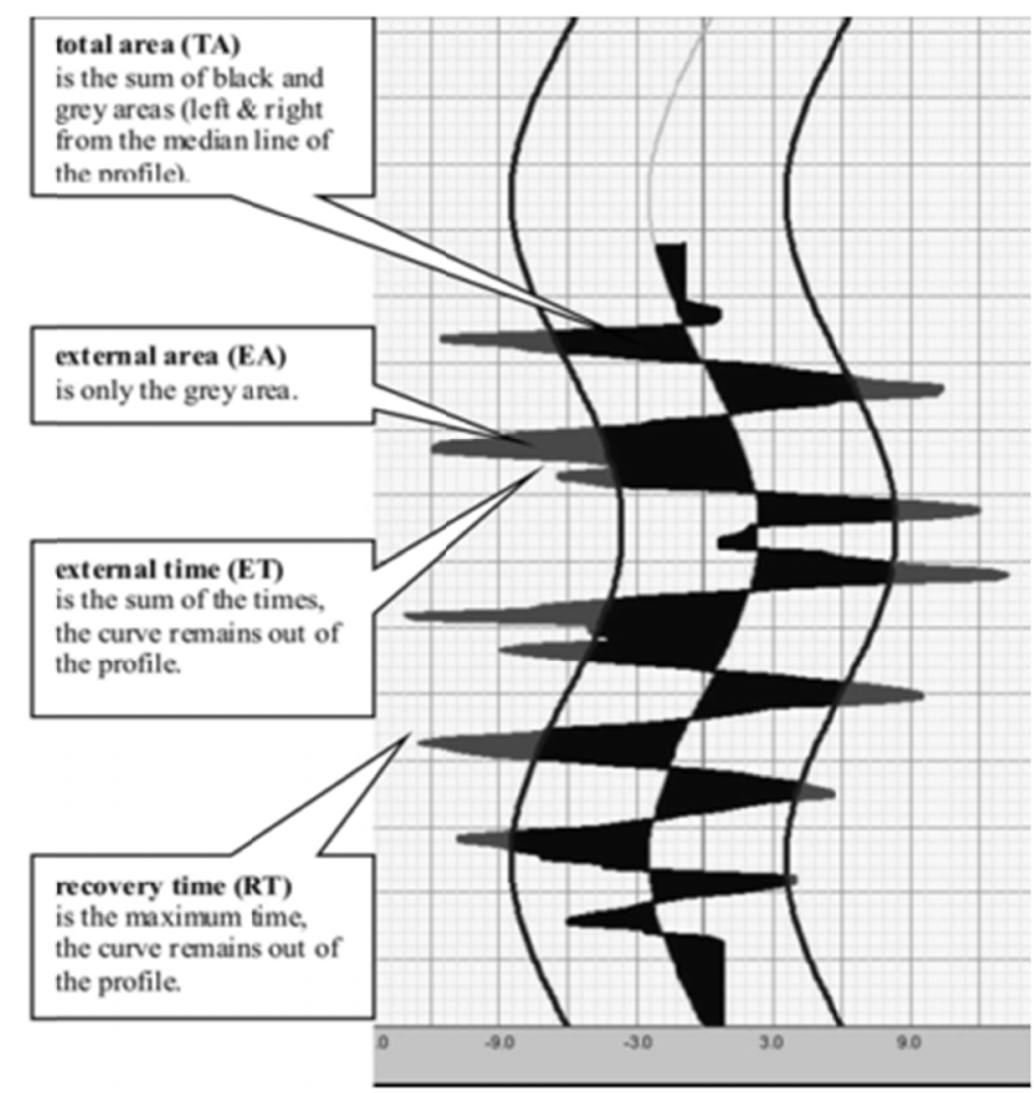

Figure 1. EasyTech Libra screenshot (Tchórzewski et al., 2013)

\subsection{Training Program}

For traditional strength training, the strength training protocol used by Tack (2013) was chosen (Tack, 2013). Squat and deadlift exercises were performed with maximum 1 repetition with $80 \%$ in $1-3$ sets and with $2-6$ repetitions in each set. 3-5 minutes rest was given between the sets. A total of 18 units of exercise were performed 3 days a week for 6 weeks. Before the training, there was 10-15 minutes dynamic warm-up. 


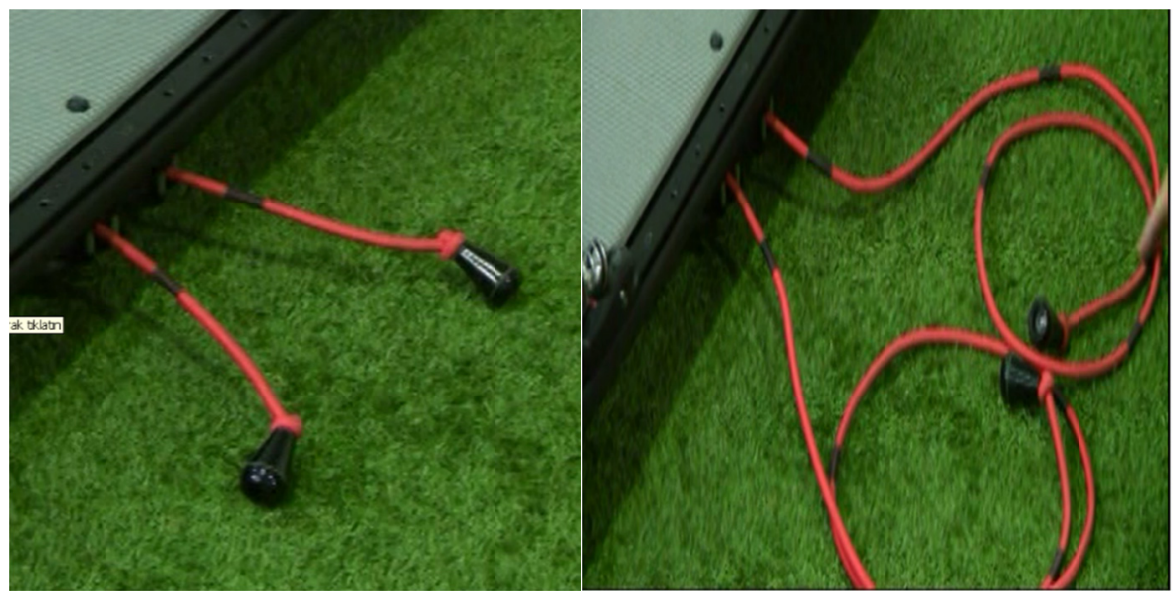

Photo 1. VertiMax resistance setting

For the VertiMax training, the basic training program recommended by the manufacturer was administered. Using the bungee cords on the VertiMax training platform, beginner level resistance program was implemented. Later, resistance levels were increased in $3 \mathrm{rd}$ and 5 th weeks according to personalized resistance levels to prepare individual training models for the participants. The manufacturing firm declares that the resistance intensity on the bungee cords will not increase jumping and landing kinematics significantly. Each bungee cord generating resistance has marked bands every 6 inches over the length of the cord. Strength is increased by pulling the marked bands on the bungee cords tied to the collars on the VertiMax to increase stretching gradually. Bungee cords allow for changes in resistance through stretching and releasing. Marked bands are effective in increasing and decreasing the level of resistance (Photo 1).

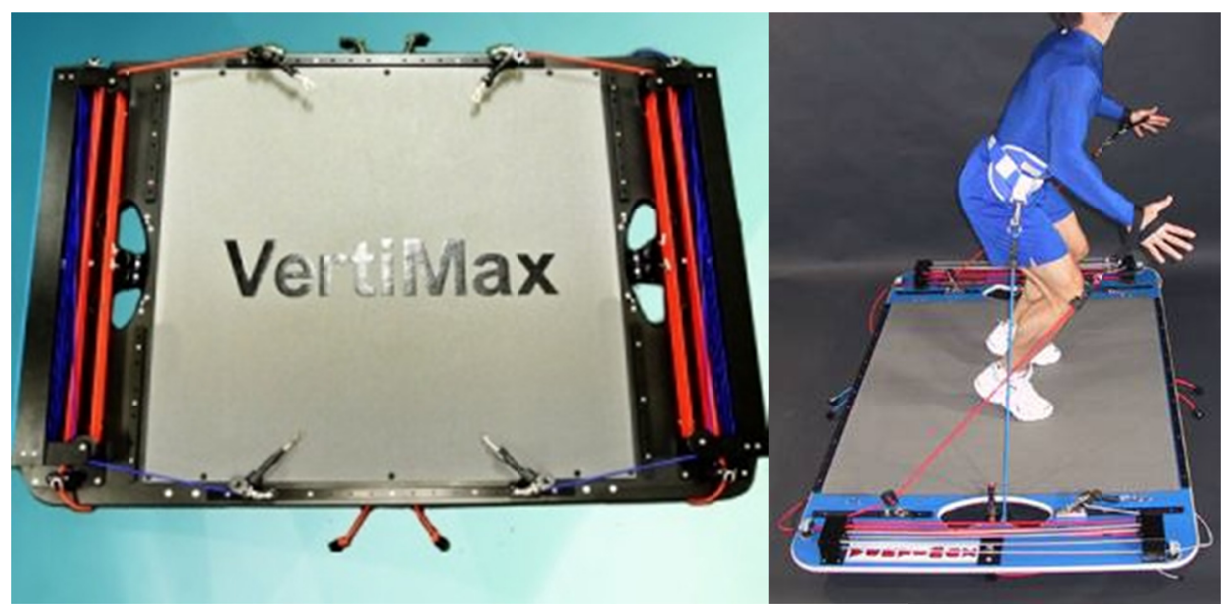

Photo 2. Vertimax and vertical jump position

Table 1. VertiMax ${ }^{\circledR}$ training protocol (Carlson et al., 2009)

\begin{tabular}{lllll}
\hline Training & Cord resistance & Resistance & Sets & Number of repetitions in sets \\
\hline Warm-up jumps & No resistance & - & 1 & 10 \\
Half squat quick jump & Bungee cord & $\geq 1$ & 2 & 10 \\
Quarter squat quick jump & Bungee cord & $\geq 1$ & 4 & 10 \\
Two-foot vertical jump & No resistance & - & 1 & 6 \\
\hline
\end{tabular}

In warm-up jumps, beginning with hands at waist level and touching the body, and bending the knees at $45^{\circ}$ to the horizontal, the subject jumped upward. As for half quick jumps, standing with hands at waist level and touching the body, and bending the knees until the thighs were parallel to the floor, the subject jumped upward, 
and returned to the starting position. Quarter and two-foot vertical jumps followed the warm-up jumps protocol while bungee cords were not used in only two-foot vertical jumps (Carlson et al., 2009, Photo 2).

\subsection{Statistical Analysis}

The three-factor mixed ANOVA (one between- and two within-subject factors) was used to assess balance levels. As descriptive statistics, mean and standard error were preferred while analyses were performed through SPSS 20 software. The level of significance was set at $\mathrm{p} \leq 0.05$.

\section{Results}

\subsection{For Balancing Scores}

Group main effect was significant, $F(50)=6.23, p=.016$. According to this finding, balance score of the classic group (26.67 \pm 0.80 ) was higher than the balance score of the VertiMax group (13.89 \pm 0.77$)$. When foot and time factors were ignored, the VertiMax group obtained better balance scores. Foot main effect was not significant $(p>.05)$. The change in balance scores was not affected by whether the subjects used right or left foot. Time main effect was significant; $F(50)=35.04, p=.000$. Accordingly, post-test balance scores $(13.99 \pm 0.60)$ were lower than pre-test balance scores $(16.57 \pm 0.60)$. Group $\mathrm{x}$ foot, group $\mathrm{x}$ time, foot $\mathrm{x}$ time and group $\mathrm{x}$ foot $\mathrm{x}$ time interaction effects were not significant ( $p>05$, Figure 2). Throughout training period, improvement in balance scores was similar for both groups and both feet.

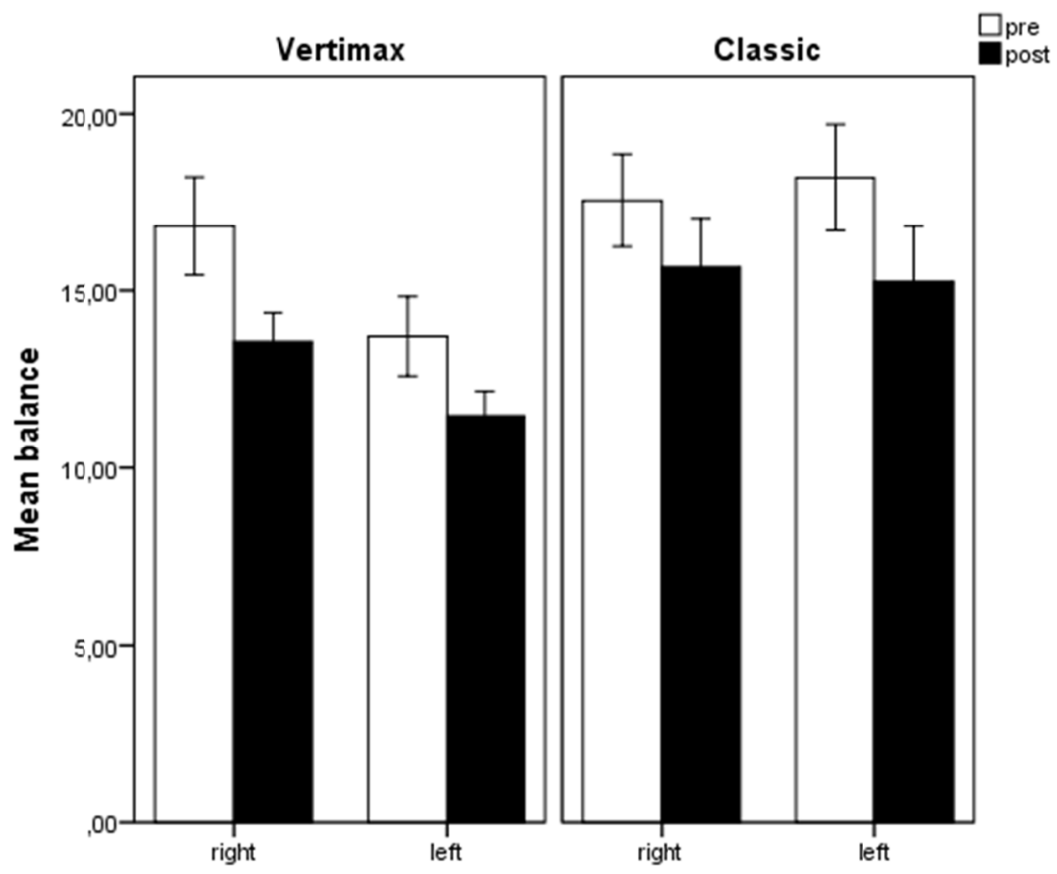

Figure 2. Balance changes in groups (+/-1 SE)

\subsection{For IBP Scores}

Group main effect was significant, $\mathrm{F}(50)=6.47, \mathrm{p}=.014$. Accordingly, IBP score of the classic group $(47.83 \pm 1.50)$ was higher than that of the VertiMax group (42.53 \pm 1.45$)$. When foot and time factors were ignored, the VertiMax group had better IBP scores. Foot main effect was not significant and the change in IBP scores was similar for both feet $(\mathrm{p}>.05)$. Time main effect was significant, $\mathrm{F}(50)=24.06, \mathrm{p}=.000$. Accordingly, post-test IBP scores $(43.13 \pm 1.12)$ were lower than pre-test IBP scores (47.24 \pm 1.12$)$. Group $\mathrm{x}$ foot, group $\mathrm{x}$ time, foot $\mathrm{x}$ time and group $\mathrm{x}$ foot $\mathrm{x}$ time interaction effects were not significant $(\mathrm{p}>.05)$ (Figure 3). Improvement in the IBP scores throughout the training was similar for both groups and both feet. 


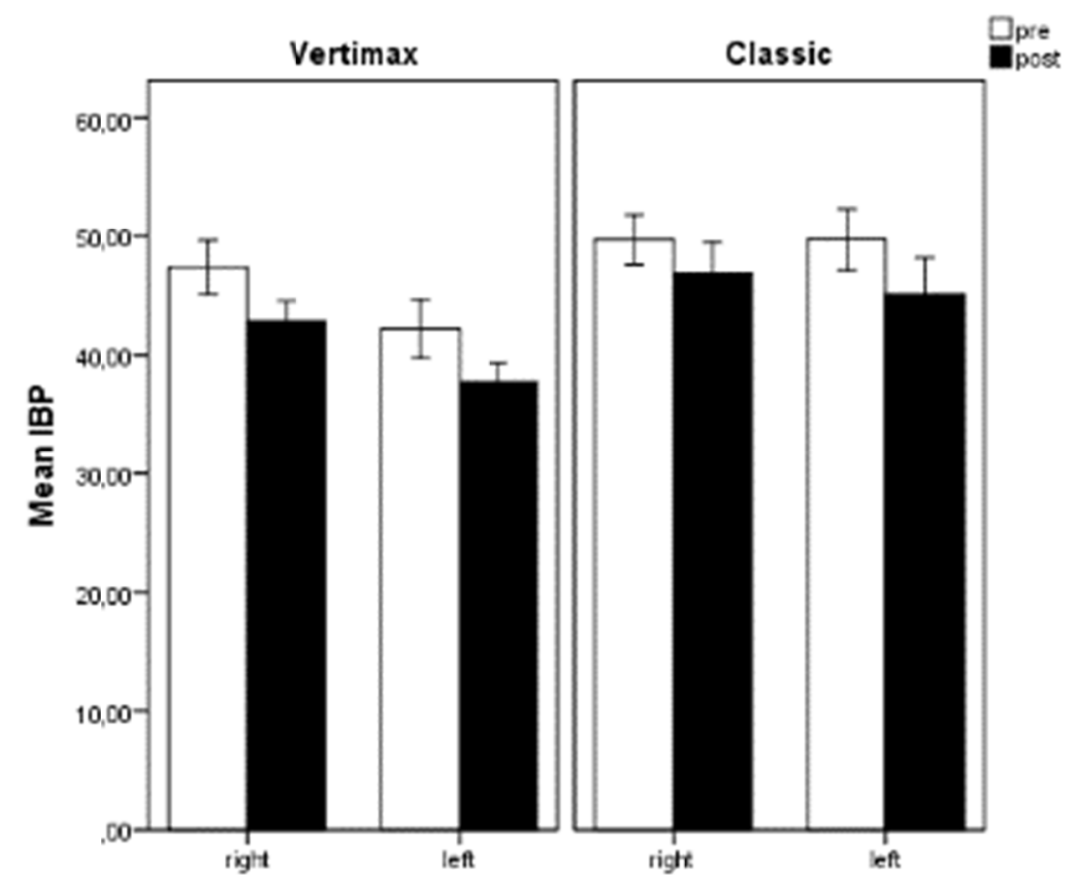

Figure 3. Changes in IBP scores (+/-1 SE)

\subsection{For Recovery Right Scores}

Group main effect was significant, $\mathrm{F}(50)=4.74, \mathrm{p}=.034$. According to this finding, recovery-right score of the classic group (1.73 \pm 0.10$)$ was higher than the recovery-right score of the VertiMax group (1.42 \pm 0.10$)$. When foot and time factors were ignored, the VertiMax group obtained better recovery-right scores. Foot main effect was significant $F(50)=5.12, p=.028$. Accordingly, right foot recovery-right scores $(1.74 \pm 0.10)$ were higher compared to left foot recovery-right scores $(1.41 \pm 0.10)$. Time main effect was significant, $\mathrm{F}(50)=12.68, \mathrm{p}=.001$. Accordingly, post-test recovery-right scores $(1.35 \pm 0.10)$ were lower than pre-test recovery-right scores $(1.79 \pm 0.10)$. This decrease indicates that returns took a shorter time. Group $\mathrm{x}$ foot, group $\mathrm{x}$ time, foot $\mathrm{x}$ time and group $\mathrm{x}$ foot $\mathrm{x}$ time interaction effects were not significant ( $\mathrm{p}>.05$ ) (Figure 4). Throughout the training period, in returns from the right side of the visual helix motion area in the EasyTech Libra computer screen, improvement in the recovery-right scores of both feet was similar for the groups and both feet. 


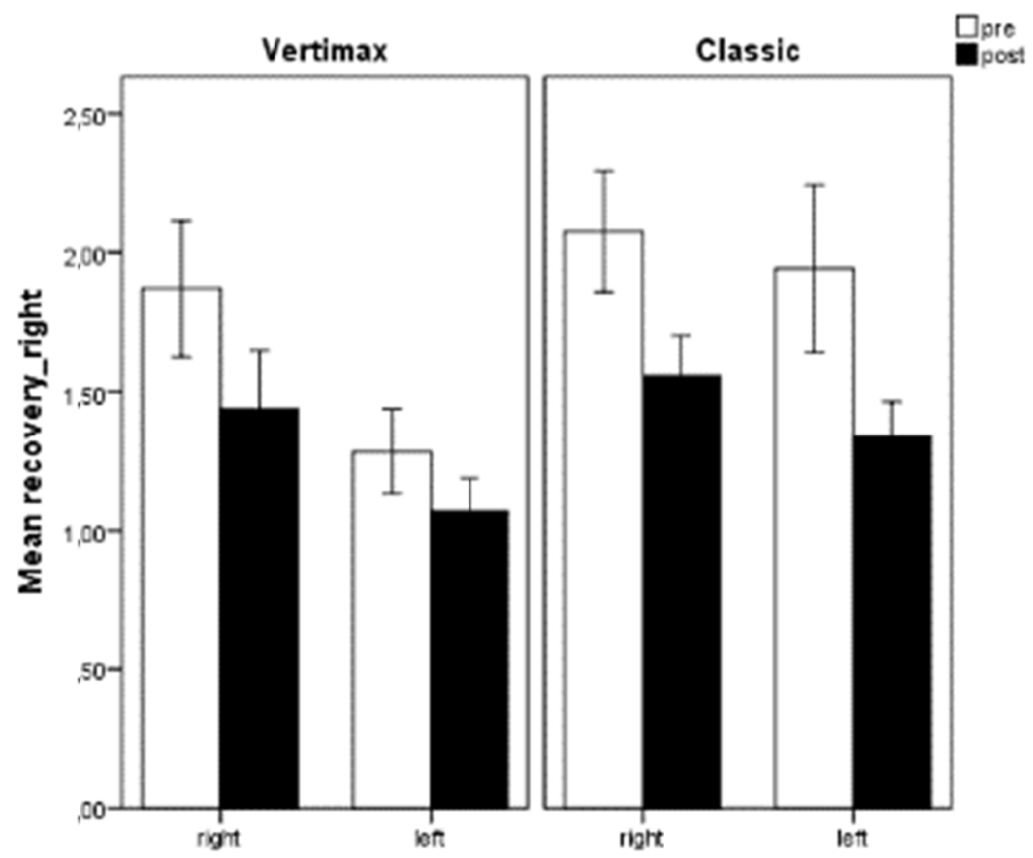

Figure 4. Changes in right foot-recovery time (+/-1 SE)

\subsection{For Recovery-Left Scores}

Group main effect was not significant $(\mathrm{p}>.05)$. Foot main effect was not significant ( $\mathrm{p}>.05)$. Time main effect was significant, $\mathrm{F}(50)=8.67, \mathrm{p}=.005$. Accordingly, post-test recovery-left scores $(1.47 \pm 0.12)$ were lower than pre-test recovery-left scores $(1.84 \pm 0.12)$. Group $\mathrm{x}$ foot, group $\mathrm{x}$ time, foot $\mathrm{x}$ time and group $\mathrm{x}$ foot $\mathrm{x}$ time interaction effects were not significant ( $\mathrm{p}>.05)$ (Figure 5). Throughout the training period, in returns from the left side of the visual helix motion area in the EasyTech Libra computer screen, improvement in the recovery-left scores of both feet was similar for the groups and both feet.

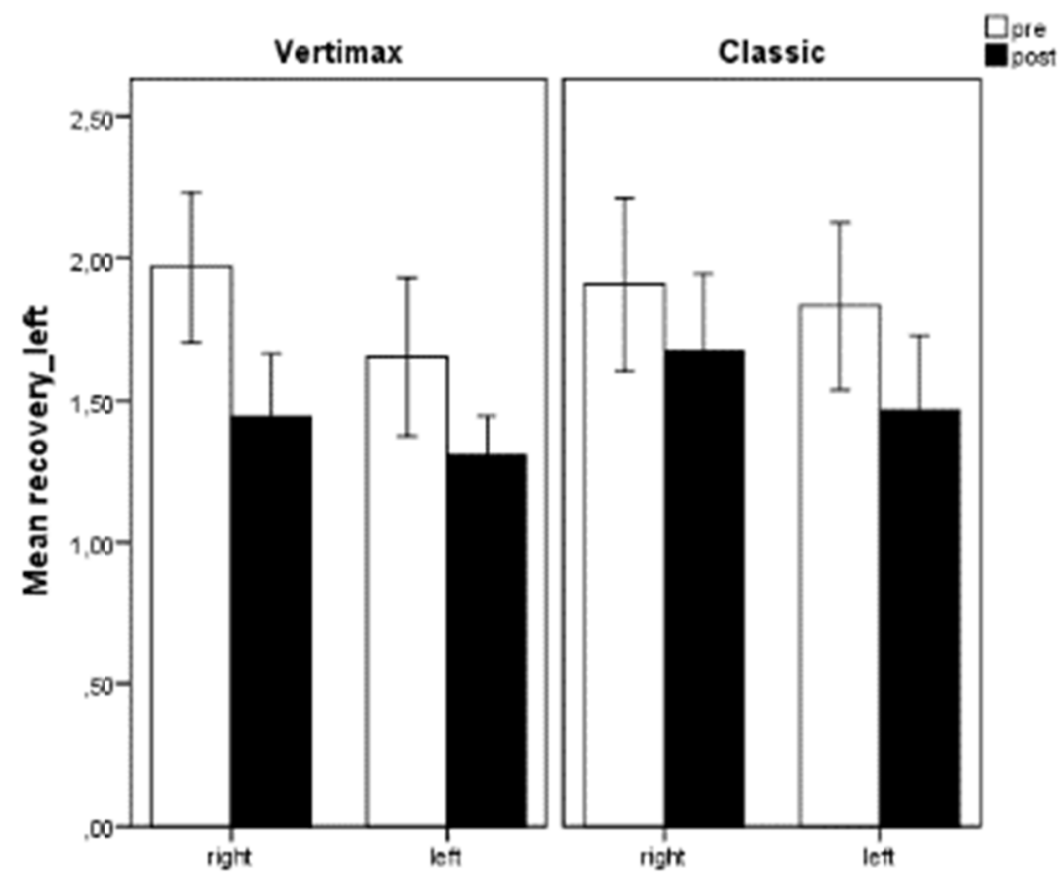

Figure 5. Changes in left foot-recovery time (+/-1 SE) 


\section{Discussion}

Decline in balance scores at the end of the training period indicates that balance was affected positively depending on potential strength development. There was a positive change in the IBP scores of the VertiMax group compared to the classic group. Depending on the training period, there was a positive change in the IBP values. This significant change in the IBP values demonstrates a decrease in balance scores, which indicates that the participants' balance ability improved and return to total area was fast. The analysis of the recovery-right scores of the training groups showed that there was a significant change in the recovery-right scores of the VertiMax group in comparison to the classic group. Also, the returns from the right side of the visual helix motion area in the EasyTech Libra computer screen were more significant in the participants in the VertiMax group compared to the classic group. Left foot values in returns from the right side of the visual helix motion area in the EasyTech Libra computer screen were more efficient than the right foot. Returns from the left side of the visual helix motion area in the EasyTech Libra computer screen showed a significant interaction at the end of the training period.

The results of the study demonstrated that each training protocol improved balance skills positively. However, the VertiMax group proved more efficient in balance development. A review of the relevant literature on the VertiMax suggests that it is described as an elastic resistance device (McClenton, 2008). It is seen that devices providing elastic resistance like the VertiMax usually develop athletes' jumping skills more than the classic methods. That is because athletes add extra load to stretch-shortening cycle that actively works during jumping (Hrysomallis, 2012). In our study, the training program administered to the VertiMax training group was similar to plyometric training in terms of kinematics.

In a study conducted in 2015, Asadi et al. examined the impact of plyometric type training on postural control and balance. The study was conducted on male basketball players. At the end of the study, Asadi et al. stated that plyometric type training had positive impacts on balance and postural control (Asadi, 2015). Similar studies in literature also indicate the positive impact of plyometric type training on balance (Myer, 2006; Arazi, 2011; Ramírez-Campillo, 2015). Movements in dynamic kinematic structures like plyometric constantly create feedback in afferent nervous system during landing, jumping and falls, improving proprioception. In this case, balance development is expected (Cronin, 2003).

Mcleod et al. analysed the effects of a 6-week neuromuscular-training program including plyometric exercises on balance motor skill. The researchers identified significant changes in participants' dynamic balance at the end of the 6-week training program. The authors stated that particularly increased proprioceptive capabilities and positive improvements in balance were related (McLeod et al., 2009). Proprioception is the ability to sense the position, location and motion of joints in space. Proprioceptive stimuli are highly important for the functioning of sensorimotor structure. Information gathered from proprioceptors is crucial for both the beginning of motion and during motion as well as in conscious movements (Lönn, 2001).

It is known that VertiMax device has positive effects on strength and power parameters particularly in lower extremity during training (Carlson et al., 2009; Rhea et al., 2008). Strength is indicated as another parameter that affects balance. Balance is a complex skill and strength is one of the basic components of the sensorimotor function supporting mobility and stabilisation (Soyuer, 2006). Balance and equilibrium are achieved primarily thanks to the muscles providing joint stability. There is a relationship between muscle strength and equilibrium (Hammami, 2016). In their study conducted in 2017, Wilson et al. found strong relationship between hip abduction strength and Y Balance Test (Wilson, 2017). In this case, balance cannot be considered independent from the strength of muscles and muscle groups creating balance.

The result of this study revealed that 2 different types of resistance training improved dynamic balance.

\section{References}

Arazi, H., \& Asadi, A. (2011). The effect of aquatic and land plyometric training on strength, sprint, and balance in young basketball players. Journal of Human Sport \& Exercise, 6(1), 101-111. https://doi.org/10.4100/jhse.2011.61.12

Asadi, A. d. (2015). The effects of plyometric type neuromuscular training on postural control performance of male team basketball players. The Journal of Strength \& Conditioning Research, 29(7), 1870-1875. https://doi.org/10.1519/JSC.0000000000000832

Bressel, E., Yonker, J. C., Kras, J., \& Heath, E. M. (2007). Comparison of static and dynamic balance in female collegiate soccer, basketball, and gymnastics athletes. Journal of Athletic Training, 42(1), 42. 
Borowski, L. A., Yard, E. E., Fields, S. K., \& Comstock, R. D. (2008). The epidemiology of US high school basketball injuries, 2005-2007. Am J Sports Med, 36(12), 2328-2335. https://doi.org/10.1177/0363546508322893

Carlson, K., Magnusen, M., \& Walters, P. (2009). Effect of various training modalities on vertical jump. Research in Sports Medicine, 17(2), 84-94. https://doi.org/10.1080/15438620902900351

Cronin, J. M. (2003). The effects of bungy weight training on muscle function and functional performance. Journal of Sports Sciences, 21(1), 59-71. https://doi.org/10.1080/0264041031000071001

Ferdjallah, M., Harris, G. F., Smith, P., \& Wertsch, J. J. (2002). Analysis of postural control synergies during quiet standing in healthy children and children with cerebral palsy. Clinical Biomechanics, 17(3), 203-210. https://doi.org/10.1016/S0268-0033(01)00121-8

Hammami, R. C. (2016). Associations between balance and muscle strength, power performance in male youth athletes of different maturity status. Pediatric Exercise Science, 28(4), 521-534. https://doi.org/10.1123/pes.2015-0231

Hahn, T., Foldspang, A., Vestergaard, E., \& Ingemann - Hansen, T. (1999). One - leg standing balance and sports activity. Scandinavian Journal of Medicine \& Science in Sports, 9(1), 15-18. https://doi.org/10.1111/j.1600-0838.1999.tb00201.x

Holcomb, W. R., Lander, J. E., Rutland, R. M., \& Wilson, G. D. (1996). The effectiveness of a modified plyometric program on power and the vertical jump. The Journal of Strength \& Conditioning Research, $10(2), 89-92$.

Hrysomallis, C. (2012). The effectiveness of resisted movement training on sprinting and jumping performance. The Journal of Strength \& Conditioning Research, 26(1), 299-306. https://doi.org/10.1519/JSC.0b013e3182185186

Kaya, M., Soyal, M., \& Karakuş, M., (2018) The effect of the leg and back strength of the serve and tennis players to the serve throwing speed and agility. Physical Education of Students, 22(5), 237-242. https://doi.org/10.15561/20755279.2018.0502

Kostopoulos, N., Bekris, E., Apostolidis, N., Kavroulakis, E., \& Kostopoulos, P. (2012). The Effect of a Balance and Proprioception Training Program on Amateur Basketball Players' Passing Skills. Journal of Physical Education and Sport, 12(3), 316-323. https://doi.org/10.7752/jpes.2012.03047

Lönn, J. (2001). Assessment of Movement and Position Sense: Methods, Theories and Applications. Thesis, Umeå University, Sweden.

McClenton, L. S. (2008). The effect of short-term VertiMax vs. depth jump training on vertical jump performance. The Journal of Strength \& Conditioning Research, 22(2), 321-325. https://doi.org10.1519/JSC.0b013e3181639f8f

McLeod, T. C. V., Armstrong, T., Miller, M., \& Sauers, J. L. (2009). Balance improvements in female high school basketball players after a 6-week neuromuscular-training program. Journal of Sport Rehabilitation, 18(4), 465-481. https://doi.org/10.1123/jsr.18.4.465

McInnes, S. E., Carlson, J. S., Jones, C. J., \& McKenna, M. J. (1995). The physiological load imposed on basketball players during competition. $J$ Sports Sci., 13(5), 387-397. https://doi.org/10.1080/02640419508732254

Myer, G. D. (2006). The effects of plyometric vs. dynamic stabilization and balance training on power, balance, and landing force in female athletes. Journal of Strength and Conditioning Research, 20(2), 345-353. https://doi.org/10.1519/R-17955.1

Paterno, M. V., Myer, G. D., Ford, K. R., \& Hewett, T. E. (2004). Neuromuscular training improves single-limb stability in young female athletes. Journal of Orthopaedic \& Sports Physical Therapy, 34(6), 305-316. https://doi.org/10.2519/jospt.2004.34.6.305

Ramírez-Campillo, R., Gallardo, F., Henriquez-Olguín, C., Meylan, C. M., Martínez, C., Álvarez, C., Caniuqueo, A., Cadore, E., \& LIzquierdo, M. (2015). Effect of vertical, horizontal, and combined plyometric training on explosive, balance, and endurance performance of young soccer players. The Journal of Strength \& Conditioning Research, 29(7), 1784-1795. https://doi.org/10.1519/JSC.0000000000000827 
Perrin, P. P., Gauchard, G. C., Perrot, C., \& Jeandel, C. (1999). Effects of physical and sporting activities on balance control in elderly people. British Journal of Sports Medicine, 33(2), 121-126. https://doi.org/10.1136/bjsm.33.2.121

Rhea, M. R., Peterson, M. D., Lunt, K. T., \& Ayllón, F. N. (2008). The effectiveness of resisted jump training on the VertiMax in high school athletes. The Journal of Strength \& Conditioning Research, 22(3), 731-734. https://doi.org/10.1519/JSC.0b013e3181660c59

Shiner, J., Bishop, T., \& Cosgarea, A. J. (2005). Integrating low-intensity plyometrics into strength and conditioning programs. Strength \& Conditioning Journal, 27(6), 10-20. https://doi.org/10.1519/00126548-200512000-00001

Soyuer, F. (2006). Relationship between lower extremity muscle strength and balance in multiple sclerosis. Journal of Neurological Sciences (Turkish), 23(4), 257-263.

Tack, C. (2013). Evidence-based guidelines for strength and conditioning in mixed martial arts. Strength \& Conditioning Journal, 35(5), 79-92. https://doi.org/10.1519/SSC.0b013e3182a62fef

Tchórzewski, D., Bujas, P., \& Jankowicz-Szymańska, A. (2013). Body posture stability in ski boots under conditions of unstable supporting surface. Journal of Human Kinetics, 38, 33-44. https://doi.org/10.2478/hukin-2013-0043

Tchórzewski, D., Jaworski, J., \& Bujas, P. (2010). Influence of long-lasting balancing on unstable surface on changes in balance. Human Movement, 11(2), 144-152. https://doi.org/10.2478/v10038-010-0022-2

Wilson, B. R. (2017). The Relationship Between Hip Strength and the Y-Balance Test. Journal of Sport Rehabilitation, 1-24. https://doi.org/10.1123/jsr.2016-0187

Young, W. B. (2006). Transfer of strength and power training to sports performance. International Journal of Sports Physiology and Performance, 1(2), 74. https://doi.org/10.1123/ijspp.1.2.74

Warren, K., Young, M. D., Jordan, D., \& Metzl, M. D. (2010). Strenght training for the young athlete. Pediatric Annals, 39(5), 293-299, https://doi.org/10.3928/00904481-20100422-10

\section{Copyrights}

Copyright for this article is retained by the author, with first publication rights granted to the journal.

This is an open-access article distributed under the terms and conditions of the Creative Commons Attribution license (http://creativecommons.org/licenses/by/4.0/). 